\title{
SIS dynamic network model based on product semantics from the perspective of infectious disease dynamics
}

\author{
Lv jiaojiao ${ }^{1, \mathrm{a}}$, Zhao yingsi ${ }^{1, \mathrm{~b}}$ \\ ${ }^{1}$ Beijing jiaotong university, No. 3, shangyuan village, haidian district, Beijing
}

\begin{abstract}
Recommendation system is unable to achive the optimal algorithm, recommendation system precision problem into bottleneck. Based on the perspective of product marketing, paper takes the inherent attribute as the classification standard and focuses on the core problem of "matching of product classification and recommendation algorithm of users' purchase demand". Three hypotheses are proposed: (1) inherent attributes of the product directly affect user demand; (2) classified product is suitable for different recommendation algorithms; (3) recommendation algorithm integration can achieve personalized customization. Based on empirical research on the relationship between characteristics of recommendation information (independent variable) and purchase intention (dependent variable), it is concluded that predictability and difference of recommendation information are not fully perceived and stimulation is insufficient. Therefore, SIS dynamic network model based on the distribution model of SIS virus is constructed. It discusses the spreading path of recommendation information and "infection" situation of consumers to enhance accurate matching of recommendation system.
\end{abstract}

\section{Introduction}

"Information explosion" is an unprecedented challenge to information providers and users. On the one hand, Information explosion is how to quickly find the information users need from massive information flow. On the other hand, it is how to provide the information accurately to users who really need [1]. Although the algorithm of electronic recommendation system is constantly optimized, the personalized definition of recommendation is difficult to achieve effective matching. Excessive product information recommendation leads to bad user experience and resource waste. This paper product categories in focus of marketing concept, build "epidemic dynamics perspective SIS dynamic network model based on product semantics", realize the customer personalization and high performance matching.

\section{Theoretical basis and literature review}

\subsection{Definition of relevant concepts}

Product classification originated from Copeland, which classified products into convenience good, shopping good and special good [2]. Verganti understood the purpose of products semantic design is to tap the potential spiritual and cultural consumer needs. Based on semantic characteristics of users' products, recommendation algorithm is applied to make personalized recommendation behavior.
Piccardi studied the interaction between disease transmission and network evolution [3]. Demirel analyzed the transmission characteristics of SIR, infectious diseases on the adaptive growth network [4]. It provides a new idea for the application of infectious diseases dynamics.

\subsection{Literature review and research on innovative points}

The existing literature mainly studies the recommendation system from two perspectives: The algorithm improvement view is adopted, but it cannot solve the problem of accuracy if it cannot propose the optimal algorithm [5]. Or adopt an extroverted user perspective to classify the characteristics of users and study the purchase needs of users. There are two shortcomings. One is the fragmentation of research. It does not combine the different requirements of users with the recommendation algorithm. Secondly, the research is loose. User demand is interfered by many factors [6]. The variability and unpredictability of human beings are the bottleneck of research.

This paper takes the inherent performance of product as research object, focuses on "matching the product classification of users' purchase needs with recommendation algorithm" [7]. The SIS dynamic network model is built. Add a product classification mechanism between the user and the algorithm of recommendation system to solve the matching problem between the diversity of users and the simplification of algorithm [8]. Finally, by studying the SIS dynamic network model under product classification, e-commerce 
realizes the accuracy and opens the "black box" between the recommendation system and user demand.

\section{Research hypothesis and model construction}

\subsection{Product classification and user requirements}

Accurate product classification is a very important dimension in the study of consumer demand and recommendation system behavior [9]. On the one hand, different kinds of products have different attributes, and consumers have different cognition and attention to them, which determines consumers' purchasing behavior. On the other hand, it affects the selection and matching of recommendation algorithm and affects the output of final recommendation results. Therefore, how to accurately define the product classification standards is the first problem.

H1: The inherent attributes of the product directly affect user requirements.

\subsection{Product classification and recommendation system}

The nodes of recommendation system network are all heterogeneous entities composed of special resource bundles, and matching enterprises and product types is a bidirectional and trial-and-error process. After the product classification, the enterprise should find recommendation algorithm network composition suitable for user's purchase demand. Therefore, how the internal recommendation algorithm match the external product classification?

$\mathrm{H} 2$ : The classified products are applicable to different recommendation algorithms.

\subsection{Recommendation system and user requirements}

Explore the mechanism of precision marketing through the network behavior of recommendation algorithm in a certain type of matching mode. Some product types tend to use the algorithm output of complementary products, while some use similar products. Therefore, the third problem is to achieve the personalized customization of output results through the behavior of algorithm in a specific product type.

H3: The integration of recommendation algorithm after classification can realize user customization.

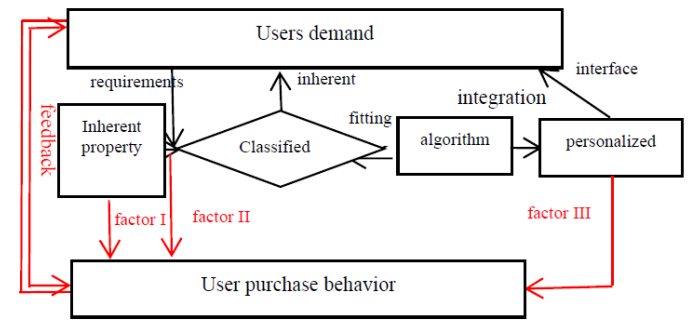

Fig1. recommendation system under product classification and feedback of user's purchasing behavior

\section{Questionnaire design and research}

\subsection{Measurement of research variables}

This paper involves the independent variable is the inherent nature of goods, the dependent variable $(\mathrm{X})$ for consumer purchase intention, it is divided into five dimensions: "consumers purchase intention" first purchase (X1), interactive (X2) and merit (X3), difference between purchase (X4), habitual purchase (X5), Independent variables inherent attribute of recommendation system under "goods" is divided into five dimensions: electronic recommendation information recognition (T1), predictive (T2), difference (T3), periodically (T4), personalized (T5).With the help of a certain scale to measure the variables and reflected in the questionnaire.

\subsection{Questionnaire design}

The questionnaire is composed of basic information, Likert-5 and Likert-7 rating scales, the scales are based on different respondents, the degree of perception from 1-7 (1-5) points are used for scoring [10]. In terms of gender, Men accounted for $40.6 \%$, the number is relatively balanced.

Table1. sample distribution

\begin{tabular}{cccc}
\hline \multicolumn{2}{c}{ Basic situation } & number & proportion \\
\hline age & $18-$ & 3 & $0.75 \%$ \\
& $18-30$ & 336 & $84.21 \%$ \\
educational & $30+$ & 60 & $15.04 \%$ \\
background & College- & 3 & $0.75 \%$ \\
& Undergraduate & 174 & $43.61 \%$ \\
Master degree & 222 & $55.64 \%$ \\
disposable & + & 33 & $8.27 \%$ \\
income & $1000-$ & 210 & $52.63 \%$ \\
& $1000-1800$ & 90 & $22.56 \%$ \\
Proportion of & $1800-2600$ & 66 & $16.54 \%$ \\
online & $2600+$ & 60 & $15.04 \%$ \\
shopping & $10 \%-$ & 111 & $27.82 \%$ \\
& $20 \%-30 \%$ & 102 & $25.56 \%$ \\
& $30 \%+$ & 126 & $31.58 \%$ \\
\hline
\end{tabular}

\section{Empirical data analysis}

\subsection{Reliability and validity test of questionnaire}

Except the difference reliability, reliability analysis coefficients of other variables are all above 0.8 , and the overall Alpha value of questionnaire reaches 0.867 , indicating the high reliability of questionnaire. In addition, KMO value is 0.853 , which means the data has validity [10]. Finally, the absolute value of factor loading coefficient is greater than 0.4 , it indicates that there is a corresponding relationship between option and factor. 
Table2. reliability and validity test of questionnaire

\begin{tabular}{|c|c|c|c|c|c|}
\hline \multirow[b]{2}{*}{ name } & \multirow{2}{*}{ CITC } & \multicolumn{2}{|c|}{ Factor load factor } & \multirow{2}{*}{$\begin{array}{c}\text { Cronbac } \\
\text { h a }\end{array}$} & \multirow[t]{2}{*}{ KMO } \\
\hline & & $\begin{array}{c}\text { Factor } \\
1\end{array}$ & Factor 2 & & \\
\hline $\begin{array}{l}\text { Recognition } \\
\text { (T1) }\end{array}$ & 0.842 & 0.840 & 0.741 & \multirow{6}{*}{0.878} & \multirow{6}{*}{0.853} \\
\hline $\begin{array}{l}\text { Predictable } \\
\quad(\mathrm{T} 2)\end{array}$ & 0.832 & 0.747 & 0.747 & & \\
\hline $\begin{array}{l}\text { Differences } \\
\quad \text { (T3) }\end{array}$ & 0.763 & 0.898 & 0.825 & & \\
\hline $\begin{array}{l}\text { Periodicity } \\
\quad(\mathrm{T} 4)\end{array}$ & 0.878 & 0.765 & 0.679 & & \\
\hline $\begin{array}{c}\text { Personalize } \\
\text { (T5) }\end{array}$ & 0.824 & 0.719 & 0.876 & & \\
\hline $\begin{array}{c}\text { Purchase } \\
\text { intention }(\mathrm{X})\end{array}$ & 0.823 & 0.754 & 0.786 & & \\
\hline
\end{tabular}

\subsection{Correlation analysis}

In this paper, consumers' purchase intention is subdivided into five categories: initial purchase, interactive purchase, selective purchase, differential purchase and habitual purchase. From the perspective of $\mathrm{T}$ (recommendation information quality), ex: $\mathrm{T} 1$ and $\mathrm{T} 2$ are significantly correlated with X1, X2, X4 and X5. From the perspective of $\mathrm{X}$ (consumer purchase intention), all the $\mathrm{X}$ factors except $\mathrm{X} 3$ are affected by all the $\mathrm{T}$ factors at $\mathrm{p}<0.05$. The recognition, predictability and difference are closely related to periodicity, while personalization is not closely related to purchase intention.

Table3. results of variable correlation analysis

\begin{tabular}{cccccc}
\hline & X1 & X2 & X3 & X4 & X5 \\
\hline T1 & $0.450^{* *}$ & $0.373 * *$ & 0.159 & $0.226^{* *}$ & $0.361^{* *}$ \\
T2 & $0.249 * *$ & $0.303 * *$ & 0.097 & $0.309^{* *}$ & $0.460^{* *}$ \\
T3 & $0.295^{* *}$ & $0.444^{* *}$ & $0.323 * *$ & $0.333^{* *}$ & $0.212^{*}$ \\
T4 & $0.197 *$ & $0.181 *$ & 0.035 & $0.203 *$ & $0.429 * *$ \\
T5 & $0.175^{*}$ & $0.317 * *$ & $0.209^{*}$ & $0.192^{*}$ & $0.331^{* *}$ \\
\hline
\end{tabular}

Initial purchase(X1): I didn't notice after being recommended.

Interactive buying(X2): The shopping behavior of others will affect my judgment.

Preferred purchase(X3): Different goods, my comparison and consideration time will be different.

Differential buying(X4): Different products, different frequency of the recommendation system.

Habitual purchase(X5): I have more desire to purchase the products recommended by the system

\subsection{Regression analysis}

This paper analyze the influence of the characteristics of commodity recommendation information (the above five aspects) on consumers' purchase intention. The influence coefficients of the predictability, periodicity and personalization on consumers' purchase intention are $0.295,0.268$ and 0.265 . In addition, the fitting of regression equation model is analyzed through the indicator R2 [11].
Table4. regression analysis results

\begin{tabular}{clcll}
\hline & \multicolumn{2}{c}{$\begin{array}{c}\text { Unstandardized } \\
\text { coefficient }\end{array}$} & R & DW value \\
\cline { 2 - 3 } & $\boldsymbol{B}$ & Standard error & & \\
\hline constant & 0.763 & 0.347 & & \\
T1 & 0.000 & 0.075 & & \\
T2 & 0.295 & 0.069 & 0.538 & 2.175 \\
T3 & 0.045 & 0.066 & & \\
T4 & 0.268 & 0.061 & & \\
T5 & 0.265 & 0.067 & & \\
\hline
\end{tabular}

Dependent variable: the recommended product completely conforms to my taste and interest $(\mathrm{X})$

\section{Build the "SIS" dynamic network model}

The individual in the terminal commodity (which can cause customer to behave a certain level of purchase behavior) is regarded as the node in the recommendation system network and each object has its own property, which is defined as the node property. Content and content between the intrinsic properties (complementary, alternative and no obvious connection.) as the network edge, then all the final goods can be abstracted as a network [12].

\subsection{Dynamics model of infectious disease}

Among them, the diseases accepted by the network (recommendation system) include: customers' completed purchase behavior, closely related purchase behavior, daily browsing behavior. In the process of disease transmission, the nodes (product attributes) in the network are in the susceptible state (S) or the infected state (l). The infected infect their susceptible neighbors via a link in the network, which includes complementary $\lambda 1$, alternative $\lambda 2$.

Table5. product classification in three dimensions

\begin{tabular}{c|c|c|c}
\hline & complementary & substitute & irrelated \\
\hline \multirow{2}{*}{$\begin{array}{c}\text { Low cost low } \\
\text { frequency }\end{array}$} & High-diversity & High-diversity & High-diversity \\
\cline { 2 - 4 } & Low-diversity & Low-diversity & Low-diversity \\
\hline $\begin{array}{c}\text { Low cost, high } \\
\text { frequency }\end{array}$ & High-diversity & High-diversity & High-diversity \\
\cline { 2 - 4 } & Low-diversity & Low-diversity & Low-diversity \\
\hline $\begin{array}{c}\text { High cost, low } \\
\text { frequency }\end{array}$ & High-diversity & High-diversity & High-diversity \\
\cline { 2 - 4 } & Low-diversity & Low-diversity & Low-diversity \\
\hline $\begin{array}{c}\text { High cost, high } \\
\text { frequency }\end{array}$ & High-diversity & High-diversity & High-diversity \\
\cline { 2 - 4 } & Low-diversity & Low-diversity & Low-diversity \\
\hline
\end{tabular}

Recommendation coefficient $\lambda:(\alpha \beta \chi \delta \varepsilon$ is constant)

$$
\lambda=\sum_{i=1}^{8} \alpha_{i}^{*}\left(c_{i}+s_{i}+i r_{i}\right)+\beta^{*}\left(\sum_{i=1}^{8} c_{i}\right)-\chi^{*}\left(\sum_{i=1}^{8} s_{i}\right)+\delta\left(\sum_{i=1}^{8} i r_{i}\right)+\varepsilon
$$

Next, we consider how to fit the recommendation coefficient and the recommendation system network after product classification. Within a sufficiently small time interval $\Delta \mathrm{t}$, the transmission process of the disease (the buying inclination and behavior that user has shown) over a dynamic network with product classification mainly 
includes four processes: contagion process, recovery process, node migration process and node migration process [13]. Each process will cause changes in delivery mode and number of different nodes types in network.

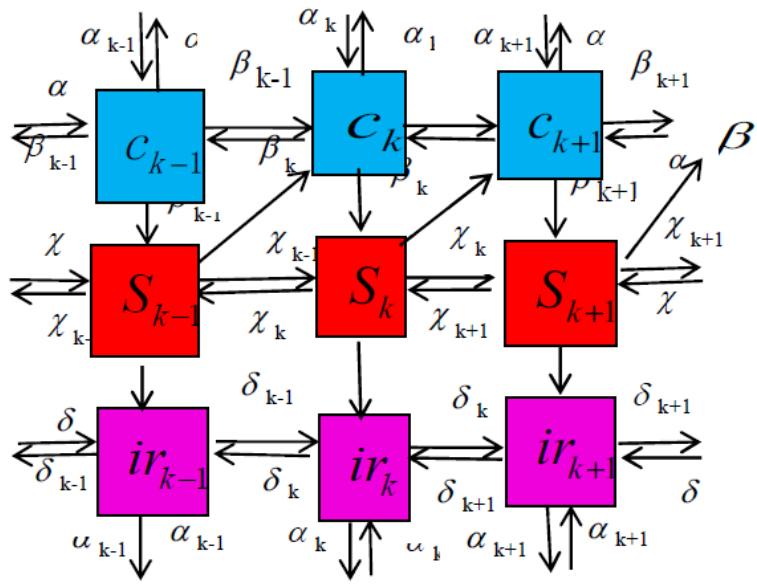

Fig2. the transmission path of the user's urchase behavior

\subsection{Summary of hypothesis test results}

Through model construction and analysis, hypothesis $\mathrm{H} 2$ made in the content of part 3 of this paper: Classified products are applicable to different recommendation algorithms, and H3: The integration of classified recommendation algorithms can achieve user customization, which is verified to be true. The summary of verification results is shown in TABLE VI.

Table6. summary of test results of research hypothesis

\begin{tabular}{ll}
\hline \multicolumn{1}{c}{ Research hypothesis } & $\begin{array}{c}\text { Inspection } \\
\text { results }\end{array}$ \\
\hline $\begin{array}{l}\text { H1: the inherent attributes of the product directly } \\
\text { affect user requirements }\end{array}$ & Set up \\
H2: the classified products are applicable to & Set up \\
different recommendation algorithms & \\
$\begin{array}{l}\text { H3: the integration of recommendation algorithm } \\
\text { after classification can realize user customization }\end{array}$ & Set up \\
\hline
\end{tabular}

\section{Conclusions and Suggestions}

\subsection{The research conclusion}

Recommendation information recognition, predictive, diversity, regular sex and personalized on consumer purchase intention have significant positive effects, but the recommendation information not fully perceived by consumers, predictability and difference the low irritating to purchase intention. This paper starting from mature SIS epidemic model, constructs a model of recommendation system under the concept of product classification, and verify its effectiveness.

\subsection{Policy Suggestions}

First, due to insufficient predictability and difference of erecommendation system, the future development direction of e-recommendation system should emphasize more on matching accuracy and intelligence. Based on the concept of product classification, pre-recommendation screening and classification can better solve this problem.

Second, the innovative pre-screening matching pattern will break through the problem of how to choose the recommendation algorithm, and help enterprises to realize the personalized customization of user recommendation. From the Angle of product marketing features and summarize product category characteristics after matching mode, explore selection mechanism behind the two sides.

Thirdly, the research on the matching mechanism between the product classification and the recommendation algorithm should be strengthened. To be specific, this paper discusses how an enterprise, after selecting a good product classification model, will act on user demand feedback through the matching behavior of the recommendation algorithm based on product classification.

\section{Acknowledgment}

Supported by "National Key R\&D Program of China" (2018YFC0832300;2018YFC0832303)

\section{References}

1. Geng bingrui. Research on personalized recommendation algorithm based on multi-objective optimization [D]. Xi 'an university of electronic science and technology, 2018.

2. Copeland, Melvin.T. The relationship of consumers buying habits to marketing methods [J]. Harvard Business Review, 1921, 1(4):282 - 289.

3. Utterback J.M., Vedin B.A., Alvare E., et al.. Design inspired Innovation[M]. Hackensack, NJ: World Scientific, 2006.

4. C. Kamp. Untangling the interplay between epidemic spread and transmission network dynamic[J]. PLoS Computational Biology. 2010, 6(11):e1000984.

5. C. Piccardi, A. Colombo, R. Casagrandi. Connectivity interplays with age in shaping contagion over networks with vital dynamics[J]. Physical Review E.2015, 91(2):022809.

6. G. Demirel, E. Barter, T. Gross. Dynamics of epidemic diseases on a growing adaptive network[J]. Scientific Reports. 2017, 7:42352.

7. liu beilin. Study on factors influencing the acceptance of e-commerce users' personalized recommendation technology [D]. China university of mining and technology (Beijing), 2009.

8. Wang rui, li peng, sun mingsong. A time-weighted network structure recommendation algorithm [J/OL]. Journal of Harbin university of science and technology, 2019 (06) : 104-108

9. Wang chuanlong, shao yabin. Hybrid recommendation system based on neighbor propagation clustering [J]. Journal of xihua university (natural science edition), 2020,39 (02) : 1-7 + 56 .

10. Chen meimei, liu limei, shi chiwei, dai weihui. Study on the influence of recommendation scale on user 
decision of personalized recommendation system [J]. Nankai management review, 2020 (01) : 180-188.

11. tong qi, liu qiang, xu saihua, hu yiguang. Research on e-commerce intelligent recommendation system based on related articles $[\mathrm{J}]$. Enterprise technology and development, 2019 (12) : 79-80.

12. jing wenjun. Study on dynamic network infectious disease model of population evolution [D]. Shanxi university, 2019

13. bao chun. Study on SARS infectious disease based on SIR model [D]. Shandong university, 2019. 